\title{
The Annual American Men's Internet Survey of Behaviors of Men Who Have Sex With Men in the United States: 2015 Key Indicators Report
}

Maria Zlotorzynska, PhD, MPH; Patrick Sullivan, PhD, DVM; Travis Sanchez, DVM, MPH

Rollins School of Public Health, Department of Epidemiology, Emory University, Atlanta, GA, United States

\section{Corresponding Author:}

Maria Zlotorzynska, $\mathrm{PhD}, \mathrm{MPH}$

Rollins School of Public Health

Department of Epidemiology

Emory University

GCR 410

1518 Clifton Rd NE

Atlanta, GA, 30322

United States

Phone: 14047278799

Fax: 14047278737

Email: $\underline{\text { maria.zlotorzynska@emory.edu }}$

\section{Abstract}

The American Men's Internet Survey (AMIS) is an annual Web-based behavioral survey of men who have sex with men (MSM) living in the United States. This Rapid Surveillance Report describes the third cycle of data collection (September 2015 through April 2016; AMIS-2015). The key indicators are the same as previously reported for AMIS (December 2013-May 2014, AMIS-2013; November 2014-April 2015, AMIS-2014). The AMIS survey methodology has not substantively changed since AMIS-2014. MSM were recruited from a variety of websites using banner advertisements and email blasts. Additionally, participants from AMIS-2014 who agreed to be recontacted for future research were emailed a link to the AMIS-2015 survey. Men were eligible to participate if they were age 15 years and older, resided in the United States, provided a valid US ZIP code, and reported ever having sex with a man. We examined demographic and recruitment characteristics using multivariable regression modeling $(P<.05)$ stratified by participants' self-reported human immunodeficiency virus (HIV) status. The AMIS-2015 round of data collection resulted in 10,217 completed surveys from MSM representing every US state and Puerto Rico. Participants were mainly non-Hispanic white, older than 40 years, living in the US South, living in urban areas, and recruited from general social networking websites. Self-reported HIV prevalence was $9.35 \%$ (955/10,217). Compared to HIV-negative/unknown status participants, HIV-positive participants were more likely to have had anal sex without a condom with any male partner in the past 12 months $(75.50 \%, 721 / 955$ vs $63.09 \%, 5843 / 9262, P<.001)$ and more likely to have had anal sex without a condom with a serodiscordant or unknown status partner $(34.45 \%, 329 / 955$ vs $17.07 \%, 1581 / 9262, P<.001)$. The reported use of marijuana and other illicit substances in the past 12 months was higher among HIV-positive participants than HIV-negative/unknown status participants (marijuana use: $24.61 \%, 235 / 955$ vs $22.96 \%, 2127 / 9262$; other illicit substance use: $28.59 \%, 273 / 955$ vs $17.51 \%, 1622 / 9262$, respectively; both $P<.001)$. Most HIV-negative/unknown status participants $(79.11 \%, 7327 / 9262)$ reported ever having a previous HIV test, and 55.69\% (5158/9262) reported HIV testing in the past 12 months. HIV-positive participants were more likely to report sexually transmitted infection (STI) testing and diagnosis compared to HIV-negative/unknown status participants (STI testing: $71.73 \%, 685 / 955$ vs $38.52 \%, 3568 / 9262$; STI diagnosis: $25.65 \%, 245 / 955$ vs $8.12 \%$, 752/9262, respectively; both $P<.001$ ).

(JMIR Public Health Surveill 2017;3(1):e13) doi: 10.2196/publichealth.7119

\section{KEYWORDS}

MSM; gay; homosexual; bisexual; HIV; STD; Internet; survey; surveillance; rapid surveillance report 


\section{Introduction}

The American Men's Internet Survey (AMIS) is an annual online behavioral survey of men who have sex with men (MSM) who live in the United States. The methods have been previously published [1,2]. This supplemental report updates that previous manuscript with the most current data available from AMIS (AMIS-2015). Methods in AMIS-2015 are unchanged from the previously published manuscript unless otherwise noted.

\section{Methods}

\section{Recruitment and Enrollment}

As in the prior year, AMIS participants were recruited through convenience sampling from a variety of websites using banner advertisements or email blasts to website members (hereafter referred to generically as "ads"). The survey was not incentivized. Data on the number of clicks on all banner ads were obtained directly from the websites. In AMIS-2014, data on the number of clicks on geospatial social networking banner ads were instead obtained by counting the number of clicks on the survey landing page. Men who clicked on the ads were taken directly to the survey website hosted on a secure server administered by SurveyGizmo (Boulder, CO, USA). Participants were also recruited by emailing participants from the previous cycle of AMIS (AMIS-2014) who consented to be recontacted for future studies. To be eligible for the survey, participants had to be 15 years of age or older, consider themselves to be male, and report that they had oral or anal sex with a man at least once in the past (hereafter referred to as MSM). Persons who reported being younger than 15 years of age or refused to provide their age were not asked any other screening questions. Those MSM who met the eligibility criteria and consented to participate in the study started the online survey immediately. The full questionnaire for AMIS-2015 is presented in Multimedia Appendix 1.

AMIS-2015 ran from September 2015 through April 2016, and resulted in 137,608 persons clicking on the ads and landing on the study's recruitment page (Table 1). Most persons who clicked on the ads were from general social networking websites $(66,500 / 137,608,48.33 \%)$. Of the 1248 participants who completed the AMIS-2014 survey and were emailed links to the AMIS-2015 survey, 9.13\% (114/1248) clicked on the link. One-third $(33.58 \%, 46,207 / 137,608)$ of those who landed on the study's page started the screening process and $56.09 \%$ $(25,919 / 46,207)$ of those were eligible. The most common reason for ineligibility was not ever having male-male sex. More than three-quarters $(78.52 \%, 20,351 / 25,919)$ of those who were eligible consented to participate in the survey. There were 2291 of $20,351(11.26 \%)$ surveys determined to likely be from duplicate participants. Deduplication of survey responses was performed in the same manner as in previous AMIS cycles [1,2]. Among unduplicated surveys, almost two-thirds (64.21\%, $11,597 / 18,060$ ) were considered successful (ie, observations with no missing values for the first question of at least two consecutive sections). Most successful surveys were among men who reported having sex with another man in the past 12 months $(89.07 \%, 10,330 / 11,597)$. Finally, 1.09\% (113/10,330) of the sample was found to have provided an invalid ZIP code and was excluded from the final analytical sample. 
Table 1. Recruitment outcomes for the American Men's Internet Survey, United States, 2015.

\begin{tabular}{|c|c|c|c|c|c|c|}
\hline \multirow[t]{2}{*}{ Recruitment outcomes } & \multirow[t]{2}{*}{ Total } & \multicolumn{5}{|l|}{ Recruitment type } \\
\hline & & $\begin{array}{l}\text { Gay social } \\
\text { networking }(\mathrm{n}=1)\end{array}$ & $\begin{array}{l}\text { General gay } \\
\text { interest }(n=2)\end{array}$ & $\begin{array}{l}\text { General social } \\
\text { networking }(n=4)\end{array}$ & $\begin{array}{l}\text { Geospatial social } \\
\text { networking }(n=2)\end{array}$ & $\begin{array}{l}\text { AMIS-2014 } \\
\text { participants }\end{array}$ \\
\hline Clicked ad, n & 137,608 & 4680 & 3968 & 66,500 & 62,261 & 199 \\
\hline Screened, ${ }^{\mathrm{a}} \mathrm{n}(\%)$ & $46,207(33.58)$ & $3671(78.44)$ & $1165(29.36)$ & $30,581(45.99)$ & $10,630(17.07)$ & $160(80.40)$ \\
\hline Ineligible, ${ }^{b}(\%)$ & $20,288(43.91)$ & $740(20.16)$ & $463(39.74)$ & $16,206(52.99)$ & $2868(26.98)$ & $11(6.88)$ \\
\hline Not age $\geq 15$ years $^{c}$ & $14,246(70.22)$ & $615(83.11)$ & $369(79.70)$ & $11,056(68.22)$ & $2197(76.60)$ & $9(81.82)$ \\
\hline Not male $e^{c}$ & $15,255(75.19)$ & $559(75.54)$ & $381(82.29)$ & $11,800(72.81)$ & $2505(87.34)$ & $10(90.91)$ \\
\hline Not ever MSM $^{\mathrm{c}}$ & $19,804(97.61)$ & $620(83.78)$ & 454 (98.06) & $16,046(99.01)$ & $2673(93.20)$ & $11(100.00)$ \\
\hline Nonresident $^{\mathrm{c}}$ & $15,502(76.41)$ & $624(84.32)$ & $377(81.43)$ & $11,469(70.77)$ & $2573(89.71)$ & $9(81.82)$ \\
\hline Eligible, ${ }^{\mathrm{b}} \mathrm{n}(\%)$ & $25,919(56.09)$ & $2931(79.84)$ & $702(60.26)$ & $14,375(47.01)$ & $7762(73.02)$ & $149(93.13)$ \\
\hline Consented, ${ }^{\mathrm{d}} \mathrm{n}(\%)$ & $20,351(78.52)$ & $2181(74.41)$ & $586(83.48)$ & $10,818(75.26)$ & $6623(85.33)$ & $143(95.97)$ \\
\hline Unduplicated, ${ }^{\mathrm{e}} \mathrm{n}(\%)$ & $18,060(88.74)$ & $2032(93.17)$ & $552(94.20)$ & $9410(86.98)$ & $5926(89.48)$ & $140(97.90)$ \\
\hline Success, ${ }^{\mathrm{f}} \mathrm{n}(\%)$ & $11,597(64.21)$ & $1568(77.17)$ & $426(77.17)$ & $6372(67.72)$ & $3104(52.38)$ & $127(90.71)$ \\
\hline MSM past 12 months, ${ }^{\mathrm{g}} \mathrm{n}(\%)$ & $10,330(89.07)$ & $1456(92.86)$ & $381(89.44)$ & $5425(85.14)$ & $2953(95.14)$ & $115(90.55)$ \\
\hline Valid ZIP code, ${ }^{\mathrm{h}} \mathrm{n}(\%)$ & $10,217(98.91)$ & 1451 (99.66) & $381(100.00)$ & $5396(99.47)$ & $2875(97.36)$ & $114(99.13)$ \\
\hline
\end{tabular}

${ }^{\text {a }}$ Proportion is of total who clicked ad. Includes those who started the screening questionnaire.

${ }^{\mathrm{b}}$ Proportion is among total screened. Ineligible includes those who did not complete the screening questionnaire.

${ }^{\mathrm{c}}$ Proportion is among total ineligible. Includes those who may not have responded to the question. MSM: men who have sex with men.

$\mathrm{d}$ Proportion is among eligible.

e Proportion is among consented. Unduplicated removes participants who were marked as duplicates using IP address and demographic data matching.

${ }^{\mathrm{f}}$ Proportion is among unduplicated. Success removes participants who did not pass the test for survey completeness.

$\mathrm{g}$ Proportion is among successes.

${ }^{\mathrm{h}}$ Proportion is among MSM in the past 12 months. Valid US ZIP codes were those that could be matched to the ZIP code-to-county crosswalk files created by the US Department of Housing and Urban Development. Any ZIP codes that could not be matched to this list were then hand-validated by checking against the ZIP code locator tool on the USPS website. ZIP codes that could not be found were classified as invalid.

Almost all these surveys $(10,217 / 10,330,98.91 \%)$ provided a valid US ZIP code. ZIP codes provided by participants were validated by merging them with the 2015 ZIP code-to-county crosswalk files created by the US Department of Housing and Urban Development [3]. Any ZIP codes that could not be matched to this list were then hand-validated by checking against the ZIP code locator tool on the United States Postal Service website [4]. ZIP codes that could not be found were classified as invalid. Overall, the completion rate was $7.4 \%$ $(10,217 / 137,608)$, with an analytical sample consisting of 10,217 surveys out of 137,068 clicks.

\section{Measures and Analyses}

For AMIS-2015 analyses, participants were categorized as either being AMIS-2014 participants who took the survey again or new participants from website/app types based on target audience and purpose: gay social networking $(n=1)$, gay general interest $(n=2)$, general social networking $(n=4)$, and geospatial social networking $(\mathrm{n}=2)$. Recruitment outcomes and demographic characteristics for the AMIS-2014 participants are presented and for all behavioral outcomes, they are recategorized according to their original recruitment source.
We do not provide the names of the websites/apps to preserve operator and client privacy, particularly where a category has only one operator. The participants who were eligible, consented, unduplicated, successful, reported male-male sex in the past 12 months, and provided a valid US ZIP code were included in analyses of participant characteristics and behavior.

For AMIS-2015, we created a more refined population density variable for each participant's county of residence as determined by their ZIP code. The levels of the population density variable are from the National Center for Health Statistics (NCHS) Rural-Urban classification scheme [5]. The NCHS classifies counties into six categories: central (ie, inner city) or fringe (ie, suburban) areas of large metropolitan statistical areas (MSAs; population size $\geq 1,000,000$ ), medium-sized MSAs (population size 250,000-999,999), small MSAs (population size <250,000), micropolitan area (counties that contain all or part of a city of 10,000 or more), and noncore (counties that do not contain any part of a city of 10,000 or more). We further collapsed these categories into a four-level urbanicity variable: urban (central), suburban (fringe), medium/small metropolitan, and rural (micropolitan and noncore). 
The analysis methods for AMIS-2015 did not substantively differ from those previously published but are repeated in this report for clarity [1]. Overall, chi-square tests were used to identify whether participant characteristics significantly differed between recruitment sources. Multivariable logistic regression modeling was used to determine significant differences in behaviors based on self-reported human immunodeficiency virus (HIV) status while controlling for race/ethnicity, age group, National HIV Behavioral System (NHBS) city residency, and recruitment website type. MSAs included in NHBS in 2015 were Atlanta, GA; Baltimore, MD; Boston, MA; Chicago, IL; Dallas, TX; Denver, CO; Detroit, MI; Houston, TX; Los Angeles, CA; Miami, FL; Nassau-Suffolk, NY; New Orleans, LA, New York City, NY; Newark, NJ; Philadelphia, PA; San Diego, CA; San Francisco, CA; San Juan, PR; Seattle, WA; and Washington, DC. Self-reported HIV status was categorized as either HIV-positive or HIV-negative/unknown status, consistent with surveillance reports produced by NHBS [6]. HIV testing behaviors were only examined among those who did not report that they were HIV-positive and were also presented by participant characteristics. Multivariable logistic regression results are presented as Wald chi-square $P$ values to denote an independently significant difference in the behavior for each subgroup compared to a referent group. Statistical significance was determined at $P<.05$.

\section{Results}

Approximately seven in $10(7291 / 10,217,71.36 \%)$ participants included in this report were white and non-Hispanic, less than half were 40 years of age or older $(4326 / 10,217,42.34 \%)$, and their most common region of residence was the South followed by the West (Table 2). Participants were recruited from all US states and there were at least 100 participants from each of 28 states (Figure 1). Overall, 9.35\% $(955 / 10,217)$ of participants reported being HIV-positive, 69.91\% (7143/10,217) reported being HIV-negative, and $20.74 \%(2119 / 10,217)$ reported having an unknown HIV status. All participant characteristics differed significantly based on where they were recruited (Table 2). 
Table 2. Characteristics of MSM participants in the American Men's Internet Survey by recruitment type, United States, 2015.

\begin{tabular}{|c|c|c|c|c|c|c|c|}
\hline \multirow[t]{2}{*}{ Participant characteristics } & \multirow[t]{2}{*}{ Total, n (\%) } & \multicolumn{5}{|c|}{ Recruitment type, n (\%) } & \multirow[t]{2}{*}{$P^{\mathrm{a}}$} \\
\hline & & $\begin{array}{l}\text { Gay social } \\
\text { networking } \\
(\mathrm{n}=1)\end{array}$ & $\begin{array}{l}\text { General gay } \\
\text { interest }(n=4)\end{array}$ & $\begin{array}{l}\text { General social } \\
\text { networking }(n=4)\end{array}$ & $\begin{array}{l}\text { Geospatial social } \\
\text { networking }(n=2)\end{array}$ & $\begin{array}{l}\text { AMIS-2014 } \\
\text { participants }\end{array}$ & \\
\hline Race/Ethnicity & & & & & & & $<.001$ \\
\hline Black, non-Hispanic & $675(6.61)$ & $33(2.27)$ & $15(3.94)$ & $444(8.23)$ & $176(6.12)$ & $7(6.14)$ & \\
\hline Hispanic & $1387(13.58)$ & $73(5.03)$ & $36(9.45)$ & 755 (13.99) & $511(17.77)$ & $12(10.53)$ & \\
\hline White, non-Hispanic & $7291(71.36)$ & $1271(87.59)$ & $301(79.00)$ & $3733(69.18)$ & $1899(66.05)$ & $87(76.32)$ & \\
\hline Other or multiple races & $864(8.46)$ & $74(5.10)$ & $29(7.61)$ & $464(8.60)$ & $289(10.05)$ & $8(7.02)$ & \\
\hline Age (years) & & & & & & & $<.001$ \\
\hline $15-24$ & $2821(27.61)$ & $32(2.21)$ & $37(9.71)$ & $2155(39.94)$ & $581(20.21)$ & $16(14.04)$ & \\
\hline $25-29$ & $1583(15.49)$ & $36(2.48)$ & $61(16.01)$ & $983(18.22)$ & $491(17.08)$ & $12(10.53)$ & \\
\hline $30-39$ & $1487(14.55)$ & $112(7.72)$ & $86(22.57)$ & $516(9.56)$ & $740(25.74)$ & $33(28.95)$ & \\
\hline$\geq 40$ & $4326(42.34)$ & $1271(87.59)$ & $197(51.71)$ & $1742(32.28)$ & $1063(36.97)$ & $53(46.49)$ & \\
\hline Region & & & & & & & .002 \\
\hline Northeast & $2038(19.95)$ & $304(20.95)$ & $72(18.90)$ & $1074(19.90)$ & $566(19.69)$ & $22(19.30)$ & \\
\hline Midwest & $2127(20.82)$ & $344(23.71)$ & $73(19.16)$ & $1152(21.35)$ & $530(18.43)$ & $28(24.56)$ & \\
\hline South & $3739(36.60)$ & $467(32.18)$ & $132(34.65)$ & $2000(37.06)$ & $1098(38.19)$ & $42(36.84)$ & \\
\hline West & $2305(22.56)$ & 335 (23.09) & $103(27.03)$ & $1166(21.61)$ & $679(23.62)$ & $22(19.30)$ & \\
\hline US dependent areas & $8(0.08)$ & $1(0.07)$ & $1(0.26)$ & $4(0.07)$ & $2(0.07)$ & $0(0.0)$ & \\
\hline NHBS city resident ${ }^{b}$ & & & & & & & $<.001$ \\
\hline Yes & $3731(36.52)$ & $565(38.94)$ & $177(46.46)$ & $1855(34.38)$ & $1090(37.91)$ & $44(38.60)$ & \\
\hline No & $6486(63.48)$ & $886(61.06)$ & $204(53.54)$ & $3541(65.62)$ & 1785 (62.09) & $70(61.40)$ & \\
\hline Population density ${ }^{\mathrm{c}}$ & & & & & & & $<.001$ \\
\hline Urban & $4101(40.18)$ & $572(39.45)$ & 189 (49.74) & $2073(38.45)$ & $1214(42.28)$ & $53(46.49)$ & \\
\hline Suburban & $2041(20.00)$ & $363(25.03)$ & $71(18.68)$ & $1092(20.26)$ & $494(17.21)$ & $21(18.42)$ & \\
\hline $\begin{array}{l}\text { Small/ medium } \\
\text { metropolitan }\end{array}$ & $3076(30.14)$ & $387(26.69)$ & $97(25.53)$ & $1679(31.14)$ & $883(30.76)$ & $30(26.32)$ & \\
\hline Rural & $988(9.68)$ & $128(8.83)$ & $23(6.05)$ & $547(10.15)$ & $280(9.75)$ & $10(8.77)$ & \\
\hline Self-reported HIV Status & & & & & & & $<.001$ \\
\hline Positive & $955(9.35)$ & $108(7.44)$ & $26(6.82)$ & $411(7.62)$ & 395 (13.74) & $15(13.16)$ & \\
\hline Negative & $7143(69.91)$ & $1102(75.95)$ & $302(79.27)$ & $3566(66.05)$ & $2080(72.35)$ & $93(81.58)$ & \\
\hline Unknown & $2119(20.74)$ & $241(16.61)$ & $53(13.91)$ & $1419(26.32)$ & $400(13.91)$ & $6(5.26)$ & \\
\hline Total & 10,217 & 1451 & 381 & 5396 & 2875 & 114 & \\
\hline
\end{tabular}

${ }^{\text {a }}$ Chi-square test for difference in characteristics between recruitment types.

${ }^{\mathrm{b}}$ NHBS: National HIV Behavioral Surveillance System.

${ }^{\mathrm{C}}$ There were 11 participants living in US territories or provided military addresses, which could not have an NCHS urban/rural category assigned.

Most participants reported having anal sex without a condom differed significantly by age group (HIV-positive and with another man within the past 12 months (Table 3). Compared to HIV-negative/unknown status participants, those who were HIV-positive were significantly more likely to report anal intercourse without a condom (adjusted OR [AOR] 1.86, 95\% CI 1.59-2.18), including with male partners who were of discordant or unknown status (AOR 2.75, 95\% CI 2.36-3.20). Within each serostatus group, anal intercourse without a condom HIV-negative/unknown status participants), and recruitment website (HIV-negative/unknown status participants only). Anal intercourse without a condom with partners of discordant or unknown HIV status differed significantly by race/ethnicity (HIV-positive participants only), recruitment website (HIV-positive participants only), and age (HIV-negative/unknown status participants only). 
Table 3. Sexual Behaviors with Male Partners of MSM Participants in the American Men's Internet Survey, United States, 2015.

\begin{tabular}{|c|c|c|c|c|c|}
\hline \multirow[t]{3}{*}{ Participant characteristics } & \multirow[t]{3}{*}{$\mathrm{n}$} & \multicolumn{4}{|c|}{ Sexual behaviors with male partners in the past 12 months } \\
\hline & & \multicolumn{2}{|c|}{ Anal intercourse without a condom } & \multicolumn{2}{|c|}{$\begin{array}{l}\text { Anal intercourse without a condom with a } \\
\text { partner of discordant or unknown HIV status }\end{array}$} \\
\hline & & $\mathrm{n}(\%)$ & $P^{\mathrm{a}}$ & $\mathrm{n}(\%)$ & $P^{\mathrm{a}}$ \\
\hline HIV positive overall & 955 & $721(75.50)$ & $<.001^{\mathrm{b}}$ & $329(34.45)$ & $<.001^{\mathrm{b}}$ \\
\hline \multicolumn{6}{|l|}{ Race/Ethnicity } \\
\hline Black, non-Hispanic & 161 & $105(65.22)$ & .08 & $35(21.74)$ & .002 \\
\hline Hispanic & 152 & $113(74.34)$ & .70 & $48(31.58)$ & .92 \\
\hline White, non-Hispanic & 573 & $454(79.23)$ & REF & $221(38.57)$ & REF \\
\hline Other or multiple races & 69 & $49(71.01)$ & .50 & $25(36.23)$ & .37 \\
\hline \multicolumn{6}{|l|}{ Age (years) } \\
\hline $15-24$ & 50 & $40(80.00)$ & .83 & $18(36.00)$ & .76 \\
\hline $25-29$ & 107 & $92(85.98)$ & .04 & $42(39.25)$ & .37 \\
\hline $30-39$ & 181 & $147(81.22)$ & .91 & $68(37.57)$ & .45 \\
\hline$\geq 40$ & 617 & $442(71.64)$ & REF & $201(32.58)$ & REF \\
\hline \multicolumn{6}{|l|}{ NHBS city resident ${ }^{c}$} \\
\hline Yes & 422 & $325(77.01)$ & .14 & $142(33.65)$ & .83 \\
\hline No & 533 & $396(74.30)$ & REF & $187(35.08)$ & REF \\
\hline \multicolumn{6}{|l|}{ Recruitment type } \\
\hline Gay social networking & 108 & $79(73.15)$ & .35 & $48(44.44)$ & .18 \\
\hline General gay interest & 26 & $22(84.62)$ & .30 & $12(46.15)$ & .37 \\
\hline General social networking & 413 & $290(70.22)$ & REF & $137(33.17)$ & REF \\
\hline Geospatial social networking & 408 & $330(80.88)$ & .59 & $132(32.35)$ & .01 \\
\hline HIV negative or unknown overall & 9262 & $5843(63.09)$ & REF & $1581(17.07)$ & REF \\
\hline \multicolumn{6}{|l|}{ Race/Ethnicity } \\
\hline Black, non-Hispanic & 514 & $316(61.48)$ & .55 & $92(17.90)$ & .44 \\
\hline Hispanic & 1235 & $804(65.10)$ & .27 & $248(20.08)$ & .05 \\
\hline White, non-Hispanic & 6718 & $4244(63.17)$ & REF & $1116(16.61)$ & REF \\
\hline Other or multiple races & 795 & $479(60.25)$ & .05 & $125(15.72)$ & .06 \\
\hline \multicolumn{6}{|l|}{ Age (years) } \\
\hline $15-24$ & 2771 & $1713(61.82)$ & $<.001$ & $524(18.91)$ & .001 \\
\hline $25-29$ & 1476 & $1072(72.63)$ & $<.001$ & $257(17.41)$ & .63 \\
\hline $30-39$ & 1306 & $930(71.21)$ & $<.001$ & $224(17.15)$ & .37 \\
\hline$\geq 40$ & 3709 & $2128(57.37)$ & REF & $576(15.53)$ & REF \\
\hline \multicolumn{6}{|l|}{ NHBS city resident ${ }^{c}$} \\
\hline Yes & 3309 & $2055(62.10)$ & .17 & $570(17.23)$ & .82 \\
\hline No & 5953 & $3788(63.63)$ & REF & $1011(16.98)$ & REF \\
\hline \multicolumn{6}{|l|}{ Recruitment type } \\
\hline Gay social networking & 1343 & $706(52.57)$ & $<.001$ & $222(16.53)$ & .49 \\
\hline General gay interest & 363 & $234(64.46)$ & .59 & $60(16.53)$ & .87 \\
\hline General social networking & 5028 & $3120(62.05)$ & REF & $816(16.23)$ & REF \\
\hline Geospatial social networking & 2528 & $1783(70.53)$ & $<.001$ & $483(19.11)$ & .06 \\
\hline
\end{tabular}


${ }^{\text {a }}$ Wald chi-square from multivariate logistic regression comparing behavior (yes vs no) among group with some characteristic compared to a referent (REF) group.

${ }^{\mathrm{b}}$ Wald chi-square from multivariate logistic regression comparing behavior (yes vs no) among HIV-positive participants compared to HIV-negative or unknown serostatus participants. Model controlled for race/ethnicity, age, NHBS residency, and recruitment type.

${ }^{\mathrm{C}}$ NHBS: National HIV Behavioral Surveillance System.

Almost one-quarter $(235 / 955,24.6 \%)$ of HIV-positive participants reported using marijuana in the past 12 months (Table 4). Compared to HIV-negative/unknown status participants, HIV-positive participants were significantly more likely to report use of marijuana (AOR 1.43, 95\% CI 1.22-1.69) and other illicit substances in the past 12 months (AOR 2.20, 95\% CI 1.88-2.59). Within each serostatus group, use of marijuana and other illicit substances differed significantly by age (HIV-positive and HIV-negative/unknown status participants), residence in an NHBS city (HIV-negative/unknown status participants only), and recruitment website type (HIV-negative/unknown status participants only). Marijuana use also differed significantly by recruitment website among HIV-positive participants. Use of other illicit substances differed significantly by race/ethnicity among HIV-negative/unknown status participants. 
Table 4. Substance using behaviors of MSM participants in the American Men's Internet Survey, United States, 2015.

\begin{tabular}{|c|c|c|c|c|c|}
\hline \multirow[t]{3}{*}{ Participant characteristics } & \multirow[t]{3}{*}{$\mathrm{n}$} & \multicolumn{4}{|c|}{ Substance use behaviors in the past 12 months } \\
\hline & & \multicolumn{2}{|c|}{ Used marijuana } & \multicolumn{2}{|c|}{ Used other substance(s) } \\
\hline & & $\mathrm{n}(\%)$ & $P^{\mathrm{a}}$ & $\mathrm{n}(\%)$ & $P^{\mathrm{a}}$ \\
\hline HIV positive overall & 955 & $235(24.61)$ & $<.001^{\mathrm{b}}$ & $273(28.59)$ & $<.001^{\mathrm{b}}$ \\
\hline \multicolumn{6}{|l|}{ Race/Ethnicity } \\
\hline Black, non-Hispanic & 161 & $40(24.84)$ & .35 & $31(19.25)$ & .06 \\
\hline Hispanic & 152 & $39(25.66)$ & .89 & $49(32.24)$ & .39 \\
\hline White, non-Hispanic & 573 & $144(25.13)$ & REF & $175(30.54)$ & REF \\
\hline Other or multiple races & 69 & $12(17.39)$ & .07 & $18(26.09)$ & 60 \\
\hline \multicolumn{6}{|l|}{ Age (years) } \\
\hline $15-24$ & 52 & $15(30.00)$ & .88 & $14(28.00)$ & .48 \\
\hline $25-29$ & 109 & $43(40.19)$ & .003 & 43 (40.19) & .02 \\
\hline 30-39 & 187 & $53(29.28)$ & .67 & $69(38.12)$ & .19 \\
\hline$\geq 40$ & 627 & $124(20.10)$ & REF & $147(23.82)$ & REF \\
\hline \multicolumn{6}{|l|}{ NHBS city resident $^{c}$} \\
\hline Yes & 422 & $110(26.07)$ & .35 & $125(29.62)$ & .45 \\
\hline No & 533 & $125(23.45)$ & REF & $148(27.77)$ & REF \\
\hline \multicolumn{6}{|l|}{ Recruitment type } \\
\hline Gay social networking & 108 & $20(18.52)$ & .89 & $28(25.93)$ & .97 \\
\hline General gay interest & 26 & $4(15.38)$ & .48 & $7(26.92)$ & .93 \\
\hline General social networking & 413 & $90(21.79)$ & REF & $95(23.00)$ & REF \\
\hline Geospatial social networking & 408 & $121(29.66)$ & .04 & $143(35.05)$ & .14 \\
\hline HIV negative or unknown overall & 9262 & $2127(22.96)$ & REF & $1622(17.51)$ & REF \\
\hline \multicolumn{6}{|l|}{ Race/Ethnicity } \\
\hline Black, non-Hispanic & 514 & $91(17.70)$ & .06 & $67(13.04)$ & .02 \\
\hline Hispanic & 1235 & $296(23.97)$ & .78 & $220(17.81)$ & .84 \\
\hline White, non-Hispanic & 6718 & $1570(23.37)$ & REF & $1200(17.86)$ & REF \\
\hline Other or multiple races & 795 & $170(21.38)$ & .32 & $135(16.98)$ & .94 \\
\hline \multicolumn{6}{|l|}{ Age (years) } \\
\hline $15-24$ & 2771 & $854(30.82)$ & $<.001$ & $543(19.60)$ & .02 \\
\hline $25-29$ & 1476 & $437(29.61)$ & $<.001$ & $356(24.12)$ & $<.001$ \\
\hline $30-39$ & 1306 & $297(22.74)$ & .16 & $254(19.45)$ & .96 \\
\hline$\geq 40$ & 3709 & $539(14.53)$ & REF & 469 (12.64) & REF \\
\hline \multicolumn{6}{|l|}{ NHBS city resident ${ }^{c}$} \\
\hline Yes & 3309 & $793(23.96)$ & .002 & $633(19.13)$ & $<.001$ \\
\hline No & 5953 & $1334(22.41)$ & REF & $989(16.61)$ & REF \\
\hline \multicolumn{6}{|l|}{ Recruitment type } \\
\hline Gay social networking & 1343 & $187(13.92)$ & .02 & $167(12.43)$ & .13 \\
\hline General gay interest & 363 & $74(20.39)$ & .92 & $57(15.70)$ & .44 \\
\hline General social networking & 5028 & $1244(24.74)$ & REF & $859(17.08)$ & REF \\
\hline Geospatial social networking & 2528 & $622(24.60)$ & .004 & $539(21.32)$ & $<.001$ \\
\hline
\end{tabular}

\footnotetext{
${ }^{\text {a }}$ Wald chi-square from multivariable logistic regression comparing behavior (yes vs no) among group with some characteristic compared to a referent 
(REF) group

${ }^{\mathrm{b}}$ Wald chi-square from multivariable logistic regression comparing behavior (yes vs no) among HIV-positive participants compared to HIV-negative or unknown serostatus participants. Model controlled for race/ethnicity, age, NHBS residency, and website type.

${ }^{\mathrm{c}}$ NHBS: National HIV Behavioral Surveillance System.

HIV testing behaviors were examined among those who did not report being HIV-positive (Table 5). Most participants $(7327 / 9262,79.11 \%)$ reported having been previously tested for HIV infection, and just over half (5158/9262, 55.69\%)

reported being tested in the past 12 months. HIV testing behavior, both ever tested and tested in past 12 months, differed significantly by age, residence in an NHBS city, and recruitment website type.

Table 5. HIV testing behaviors of HIV-negative or unknown status MSM participants in the American Men's Internet Survey, United States, 2015.

\begin{tabular}{|c|c|c|c|c|c|}
\hline \multirow[t]{3}{*}{ Participant characteristics } & \multirow[t]{3}{*}{$\mathrm{n}$} & \multicolumn{4}{|c|}{ HIV testing behaviors } \\
\hline & & \multicolumn{2}{|c|}{ HIV tested ever } & \multicolumn{2}{|c|}{ HIV tested past 12 months } \\
\hline & & $\mathrm{n}(\%)$ & $P^{\mathrm{a}}$ & $\mathrm{n}(\%)$ & $P^{\mathrm{a}}$ \\
\hline \multicolumn{6}{|l|}{ Race/Ethnicity } \\
\hline Black, non-Hispanic & 514 & $445(86.58)$ & .06 & $333(64.79)$ & .02 \\
\hline Hispanic & 1235 & $948(76.76)$ & .35 & $715(57.89)$ & .37 \\
\hline White, non-Hispanic & 6718 & $5314(79.10)$ & REF & $3645(54.26)$ & REF \\
\hline Other or multiple races & 795 & $620(77.99)$ & .99 & $465(58.49)$ & .81 \\
\hline \multicolumn{6}{|l|}{ Age (years) } \\
\hline $15-24$ & 2771 & $1599(57.70)$ & $<.001$ & $1286(46.41)$ & $<.001$ \\
\hline $25-29$ & 1476 & $1269(85.98)$ & $<.001$ & $903(61.18)$ & $<.001$ \\
\hline $30-39$ & 1306 & $1160(88.82)$ & $<.001$ & $858(65.70)$ & $<.001$ \\
\hline 40 or older & 3709 & $3299(88.95)$ & REF & $2111(56.92)$ & REF \\
\hline \multicolumn{6}{|l|}{ NHBS city resident ${ }^{b}$} \\
\hline Yes & 3309 & $2774(83.83)$ & $<.001$ & $2075(62.71)$ & $<.001$ \\
\hline No & 5953 & $4553(76.48)$ & REF & 3083 (51.79) & REF \\
\hline \multicolumn{6}{|l|}{ Recruitment type } \\
\hline Gay social networking & 1343 & $1122(83.54)$ & $<.001$ & $713(53.09)$ & .005 \\
\hline General gay interest & 363 & $311(85.67)$ & .87 & $189(52.07)$ & .002 \\
\hline General social networking & 5028 & $3694(73.47)$ & REF & $2512(49.96)$ & REF \\
\hline Geospatial social networking & 2528 & $2200(87.03)$ & $<.001$ & $1744(68.99)$ & $<.001$ \\
\hline Total & 9262 & $7327(79.11)$ & & $5158(55.69)$ & \\
\hline
\end{tabular}

${ }^{a}$ Wald chi-square from multivariable logistic regression comparing behavior (yes vs no) among group with some characteristic compared to a referent (REF) group.

${ }^{\mathrm{b}}$ NHBS: National HIV Behavioral Surveillance System.

Compared to HIV-negative/unknown status participants, HIV-positive participants were significantly more likely to report sexually transmitted infection (STI) testing (AOR 4.00, 95\% CI 3.43-4.68) and STI diagnosis (AOR 3.83, 95\% CI 3.20-4.59) in the past 12 months (Table 6). The most common STI diagnosis among HIV-positive participants was syphilis $(144 / 955,15.1 \%)$, whereas gonorrhea was the most common STI diagnosis among HIV-negative/unknown status participants
(427/9262, 4.61\%). Among HIV-negative/unknown status participants, STI testing differed significantly by race/ethnicity, age, and residence in an NHBS city. Among both HIV-positive and HIV-negative/unknown status participants, STI testing differed significantly by recruitment website type and STI diagnosis differed significantly by age, NHBS city residence, and recruitment website type. 
Table 6. Sexually transmitted infection testing and diagnosis of MSM participants in the American Men's Internet Survey, United States, 2015.

\begin{tabular}{|c|c|c|c|c|c|}
\hline \multirow[t]{3}{*}{ Participant characteristics } & \multirow[t]{3}{*}{$\mathrm{n}$} & \multicolumn{4}{|c|}{ STI History in the Past 12 Months } \\
\hline & & \multicolumn{2}{|c|}{ Tested for any $\mathrm{STI}^{\mathrm{a}}$} & \multicolumn{2}{|c|}{ Diagnosed with any STI ${ }^{\mathrm{a}}$} \\
\hline & & $\mathrm{n}(\%)$ & $P^{\mathrm{b}}$ & $\mathrm{n}(\%)$ & $P^{\mathrm{b}}$ \\
\hline HIV positive overall & 955 & $685(71.73)$ & $<.001^{\mathrm{c}}$ & $245(25.65)$ & $<.001^{\mathrm{c}}$ \\
\hline \multicolumn{6}{|l|}{ Race/Ethnicity } \\
\hline Black, non-Hispanic & 161 & $116(72.05)$ & .39 & $48(29.81)$ & .10 \\
\hline Hispanic & 152 & $109(71.71)$ & .29 & $50(32.89)$ & .75 \\
\hline White, non-Hispanic & 573 & $413(72.08)$ & REF & $130(22.69)$ & REF \\
\hline Other or multiple races & 69 & $47(68.12)$ & .32 & $17(24.64)$ & .25 \\
\hline \multicolumn{6}{|l|}{ Age (years) } \\
\hline $15-24$ & 50 & $44(88.00)$ & .09 & $23(46.00)$ & .02 \\
\hline $25-29$ & 107 & $89(83.18)$ & .45 & $43(40.19)$ & .09 \\
\hline $30-39$ & 181 & $152(83.98)$ & .56 & $66(36.46)$ & .77 \\
\hline$\geq 40$ & 617 & $400(64.83)$ & REF & $113(18.31)$ & REF \\
\hline \multicolumn{6}{|l|}{ NHBS city resident ${ }^{d}$} \\
\hline Yes & 422 & $313(74.17)$ & .10 & $128(30.33)$ & .007 \\
\hline No & 533 & $372(69.79)$ & REF & $117(21.95)$ & REF \\
\hline \multicolumn{6}{|l|}{ Recruitment website type } \\
\hline Gay social networking & 108 & $71(65.74)$ & .81 & $21(19.44)$ & .40 \\
\hline General gay interest & 26 & $15(57.69)$ & .25 & $3(11.54)$ & .26 \\
\hline General social networking & 413 & $276(66.83)$ & REF & $79(19.13)$ & REF \\
\hline Geospatial social networking & 408 & $323(79.17)$ & .006 & $142(34.80)$ & .003 \\
\hline HIV negative or unknown overall & 9262 & $3568(38.52)$ & REF & $752(8.12)$ & REF \\
\hline \multicolumn{6}{|l|}{ Race/Ethnicity } \\
\hline Black, non-Hispanic & 514 & $241(46.89)$ & .01 & $57(11.09)$ & .07 \\
\hline Hispanic & 1235 & $543(43.97)$ & .92 & $150(12.15)$ & .04 \\
\hline White, non-Hispanic & 6718 & $2458(36.59)$ & REF & $481(7.16)$ & REF \\
\hline Other or multiple races & 795 & $326(41.01)$ & .30 & $64(8.05)$ & .03 \\
\hline \multicolumn{6}{|l|}{ Age (years) } \\
\hline $15-24$ & 2771 & $997(35.98)$ & $<.001$ & $229(8.26)$ & .46 \\
\hline $25-29$ & 1476 & $753(51.02)$ & $<.001$ & $169(11.45)$ & $<.001$ \\
\hline $30-39$ & 1306 & $639(48.93)$ & .004 & $154(11.79)$ & .15 \\
\hline$\geq 40$ & 3709 & $1179(31.79)$ & REF & $200(5.39)$ & REF \\
\hline \multicolumn{6}{|l|}{ NHBS city resident ${ }^{c}$} \\
\hline Yes & 3309 & $1493(45.12)$ & $<.001$ & $350(10.58)$ & $<.001$ \\
\hline No & 5953 & $2075(34.86)$ & REF & $402(6.75)$ & REF \\
\hline \multicolumn{6}{|l|}{ Recruitment website type } \\
\hline Gay social networking & 1343 & $365(27.18)$ & $<.001$ & $59(4.39)$ & .04 \\
\hline General gay interest & 363 & $143(39.39)$ & .88 & $23(6.34)$ & .27 \\
\hline General social networking & 5028 & $1746(34.73)$ & REF & $313(6.23)$ & REF \\
\hline Geospatial social networking & 2528 & $1314(51.98)$ & $<.001$ & $357(14.12)$ & $<.001$ \\
\hline
\end{tabular}

\footnotetext{
${ }^{a}$ Sexually transmitted infection (STI) includes chlamydia, gonorrhea, and syphilis.
} 
${ }^{\mathrm{b}}$ Wald chi-square from multivariable logistic regression comparing behavior (yes vs no) among group with some characteristic compared to a referent (REF) group.

${ }^{\mathrm{c}}$ Wald chi-square from multivariable logistic regression comparing behavior (yes vs no) among HIV-positive participants compared to HIV-negative or unknown serostatus participants. Model controlled for race/ethnicity, age, NHBS residency, and website type.

${ }^{\mathrm{d}}$ NHBS: National HIV Behavioral Surveillance System.

Figure 1. Number of MSM participants in the American Men's Internet Survey by state, 2015.

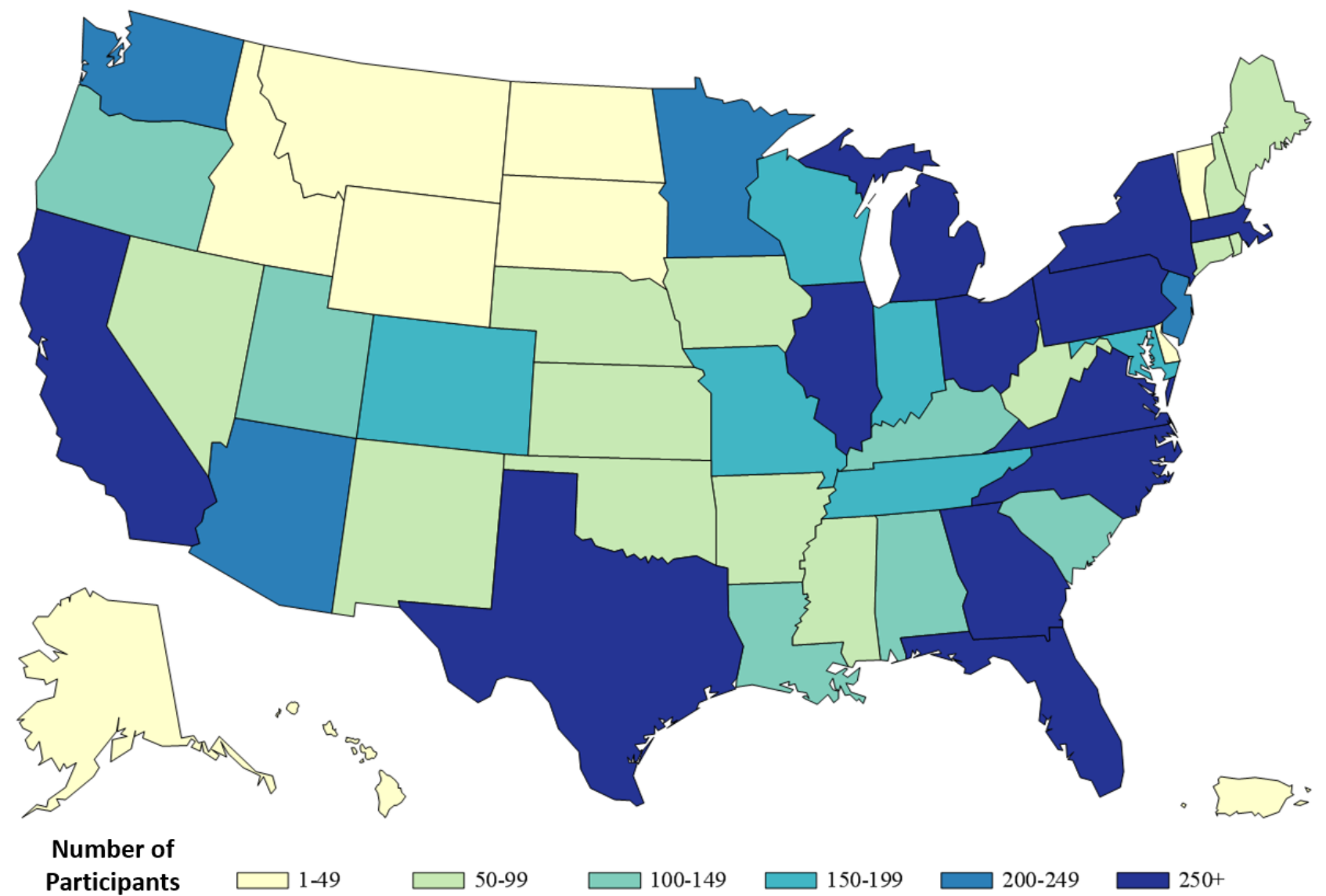

\section{Acknowledgments}

The study was funded by a grant from the MAC AIDS Fund and by the National Institutes of Health (P30AI050409)—the Emory Center for AIDS Research.

\section{Conflicts of Interest}

Authors Sanchez and Sullivan are members of the Editorial Board of JMIR Public Health and Surveillance. However, they had no involvement in the editorial decision for this manuscript. It was reviewed and handled by an independent editor.

\section{Multimedia Appendix 1}

AMIS 2015 Questionnaire.

[PDF File (Adobe PDF File), 452KB-Multimedia Appendix 1]

\section{Multimedia Appendix 2}

Recruitment and enrollment outcomes flow chart.

[PPTX File, 43KB-Multimedia Appendix 2]

\section{References}


1. Sanchez T, Zlotorzynska M, Sineath C, Kahle E, Sullivan P. The annual American Men's Internet Survey of Behaviors of Men Who Have Sex With Men in the United States: 2014 key indicators report. JMIR Public Health Surveill 2016 May 25;2(1):e23 [FREE Full text] [doi: 10.2196/publichealth.5476] [Medline: 27244770]

2. Sanchez TH, Sineath RC, Kahle EM, Tregear SJ, Sullivan PS. The annual American Men's Internet Survey of Behaviors of Men Who Have Sex With Men in the United States: protocol and key indicators report 2013. JMIR Public Health Surveill 2015;1(1):e3 [FREE Full text] [doi: 10.2196/publichealth.4314] [Medline: 27227126]

3. Office of Policy Development and Research (PD\&R), US Department of Housing and Urban Development. HUD USPS ZIP code crosswalk files URL: https://www.huduser.gov/portal/datasets/usps crosswalk.html [accessed 2017-03-02] [WebCite Cache ID 6ofllime9]

4. USPS. ZIP code lookup URL: https://tools.usps.com/go/ZipLookupAction_input [accessed 2017-03-02] [WebCite Cache ID 6oflqHEXV]

5. Ingram DD, Franco SJ. 2013 NCHS urban-rural classification scheme for counties. Vital Health Stat 2 2014 Apr(166):1-73 [FREE Full text] [Medline: 24776070]

6. Centers for Disease Control and Prevention. 2016. HIV surveillance special report 15: HIV infection risk, prevention, and testing behaviors among men who have sex with men, 2014 URL: http://www.cdc.gov/hiv/library/reports/surveillance/ \#panel2 [accessed 2017-03-02] [WebCite Cache ID 6oflxZj1a]

\author{
Abbreviations \\ AMIS: American Men's Internet Survey \\ HIV: human immunodeficiency virus \\ MSA: Metropolitan Statistical Area \\ MSM: men who have sex with men \\ NCHS: National Center for Health Statistics \\ NHBS: National HIV Behavioral Surveillance System \\ STI: sexually transmitted infection
}

\author{
Edited by G Eysenbach; submitted 07.12.16; peer-reviewed by C Khosropour, N Lachowsky; comments to author 05.01.17; revised \\ version received 24.01.17; accepted 08.02.17; published 25.03.17 \\ Please cite as: \\ Zlotorzynska M, Sullivan P, Sanchez T \\ The Annual American Men's Internet Survey of Behaviors of Men Who Have Sex With Men in the United States: 2015 Key Indicators \\ Report \\ JMIR Public Health Surveill 2017;3(1):e13 \\ URL: http://publichealth.jmir.org/2017/1/e13/ \\ doi: 10.2196/publichealth.7119 \\ PMID: 28356240
}

CMaria Zlotorzynska, Patrick Sullivan, Travis Sanchez. Originally published in JMIR Public Health and Surveillance (http://publichealth.jmir.org), 25.03.2017. This is an open-access article distributed under the terms of the Creative Commons Attribution License (http://creativecommons.org/licenses/by/2.0/), which permits unrestricted use, distribution, and reproduction in any medium, provided the original work, first published in JMIR Public Health and Surveillance, is properly cited. The complete bibliographic information, a link to the original publication on http://publichealth.jmir.org, as well as this copyright and license information must be included. 